\title{
Acciones Cooperativas que Contribuyan a la Valoración Ambiental del Entorno Educativo
}

Autora: Carmen Elena García Moreno Universidad Pedagógica Experimental Libertador, UPEL jesmarbalni@gmail.com Barinas, Venezuela

\section{Resumen}

La presente investigación tiene como objetivo general promover acciones cooperativas que contribuyan a la valoración ambiental del entorno educativo en el Centro de Educación Inicial Bolivariano "Cinqueña III" del municipio Barinas estado Barinas. El modelo de la investigación fue cualitativo, el método es investigación acción. Los informantes claves son cinco (5) docentes, veintidós (22) padres y representantes; así como veintiséis (26) niños y niñas del mencionado centro educativo, los cuales fueron seleccionados porque pertenecen al mismo contexto de estudio. Las técnicas que se utilizarán para la recopilación de la información serán la observación directa y por ello se construirán una guía de observación dirigida a los padres y representantes, para los docentes se utilizará la entrevista, por tal razón se diseñará un guión de entrevista a ser aplicados a los mismos. Además, se tomará como validez y fiabilidad de los instrumentos el proceso de triangulación, el cual, implica que los datos se recojan desde variados puntos de vistas, que permitan comparaciones diversas a un mismo fenómeno, de un grupo en varios momentos; de esta manera aumenta el grado de validación de los resultados obtenidos, permitiendo llevar a la práctica los valores que amplían la conciencia ambiental en los infantes. Debido a esto se afirma que las acciones cooperativas es una estrategia de gran cuantía para fortalecer los valores ambientales. educativo.

Palabras clave: acciones cooperativas; valoración ambiental; entorno

Fecha de Recepción: 16-11-2016

Fecha de Aceptación: 11-01-2017 


\title{
Cooperative Actions Contributing to the Environmental Assessment of the Educational Environment
}

\begin{abstract}
The present research has as general objective to promote cooperative actions that contribute to the environmental valuation of the educational environment in the Center of Initial Education Bolivariano "Cinqueña III" of the municipality Barinas state Barinas. The research model was qualitative, the method is action research. The key informants are five (5) teachers, twentytwo (22) parents and representatives; as well as twenty-six (26) boys and girls of the mentioned educational center, who were selected because they belong to the same context of study. The techniques that will be used to compile the information will be direct observation and for that reason an observation guide will be built for parents and representatives, for the teachers the interview will be used, for that reason an interview script will be designed to be Applied to them. In addition, the triangulation process will be taken as validity and reliability of the instruments, which implies that the data are collected from various points of view, allowing diverse comparisons to the same phenomenon, of a group at several moments; In this way increases the degree of validation of the results obtained
\end{abstract}

Keywords: cooperative actions; environmental valuation; educational environment.

Date Received: 16-11-2016

Date Acceptance: 11-01-2017 


\section{Introducción}

La problemática ambiental, es sin duda el problema de primer orden en la actualidad, porque se inserta en los modelos educativos como parte integral de la información que han de ser transmitidos a los niños y niñas de los diferentes niveles del Sistema Educativo, Ante esta realidad, es vital que se ofrezcan alternativas de ambiente para que los educandos mejoren su calidad de vida a través de la promoción de acciones cooperativas que contribuya a la valoración ambiental del entorno educativo en el Centro de Educación Inicial Bolivariano "Cinqueña III" del municipio Barinas del estado Barinas. El estudio se desarrolló bajo un enfoque cualitativo basado en un diseño de campo y tipo investigación acción.

\section{EI Problema}

En Venezuela esta situación fundamentada por el Grupo Ecológico Ozono (2006), muestra además que el derecho a la vida debe ser entendido como el derecho a una vivencia aceptable, como resultado de la armonía lograda en todos los aspectos que inciden en el bienestar y salud del individuo.

Sin embargo, los daños causados por el hombre a la naturaleza con el indiscriminado actuar, los desequilibrios ambientales que ocasiona han contribuido al deterioro considerable de la calidad de vida de los ciudadanos y ciudadanas. Asimismo, ese deterioro al ambiente afecta el nivel de vida de la población, arruina espacios públicos para la recreación y limita las posibilidades de idear formas alternativas de subsistencia, lo que debe ser una preocupación colectiva, así como buscar alternativas viables para la recuperación del ambiente.

En este escenario, Cárdenas (2007), señala que "la educación es la base de la formación y preparación de los recursos humanos necesarios" (pág. 4). Por ello, la escuela se convierte así en el lugar para la adquisición y difusión de los conocimientos relevantes y el medio para la multiplicación de las 
capacidades productivas. Esto justifica que en la sociedad actual es compleja y cambiante, por lo tanto al sistema educativo se le exige, cada vez más, nuevas orientaciones en sus contenidos y enseñanzas acordes a los cambios económicos, científicos y tecnológicos.

Siendo así, mediante la educación se busca la formación de seres activos en la solución de los problemas, se demandan cambios de pensamiento y de conducta, se intenta formar hombres y mujeres diferentes.

Entre los aspectos antes mencionados cabe destacar las siguientes interrogantes: ¿Cuáles son las necesidades existentes en cuanto la valoración ambiental del entorno educativo en el Centro de Educación Inicial Bolivariano "Cinqueña III"?

¿Cuáles son las acciones cooperativas que contribuyen a la valoración ambiental del entorno educativo en el Centro de Educación Inicial Bolivariano "Cinqueña III" del municipio Barinas del estado Barinas?

\subsection{Objetivos de Investigación}

\subsubsection{Objetivo General}

Promover acciones cooperativas que contribuyen a la valoración ambiental del entorno educativo en el Centro de Educación Inicial Bolivariano "Cinqueña III" del municipio Barinas del estado Barinas.

\section{Marco referencial}

\subsection{Antecedentes de la investigación}

Se refiere a los estudios previos y tesis de grado relacionadas con el problema planteado, es decir, investigaciones realizadas anteriormente y que guardan alguna vinculación con el problema en estudio, Se incluyen aquí, las opiniones, conclusiones y recomendaciones realizadas por otros autores que han tratado la problemática que constituye el núcleo, centro u objeto de la investigación que se ha abordado. 
Según Hernández, Fernández y Baptista (2010) consiste en "el desarrollo de dos etapas propiamente dichas; la revisión de la literatura y la adopción de una teoría o desarrollo de una perspectiva teórica" (pág. 23) Por otra parte, implica la revisión de estudios previos al problema, se deben señalar, además de los autores y el año en que se realizaron los estudios, los objetivos y principales hallazgos de los mismos. Aunque los antecedentes constituyen elementos teóricos, éstos pueden preceder a los objetivos, ya que su búsqueda es una de las primeras actividades que debe realizar el tesista, lo que le permitirá precisar y delimitar el objeto de estudio y por consiguiente los propósitos de la investigación.

\subsection{Bases Teóricas}

Comprenden un conjunto de conceptos y proposiciones que constituyen un punto de vista o enfoque determinado, dirigido a explicar el fenómeno $o$ problema planteado. Esta sección puede dividirse en función de los tópicos que integran la temática tratada o de las variables que serán analizadas. En tal sentido, Hernández, Fernández y Baptista (2010) expresan "una vez que se ha definido el problema y redactado los objetivos, se esquematizan los temas que deben ser desarrollados, para exponer los rasgos más relevantes de la investigación, sustentados en basamentos teóricos" (pág. 85)

\section{Acciones Cooperativas}

Las acciones cooperativas en la relación escuela comunidad, constituyen en el marco de la ejecución una herramienta esencial donde los actores con el apoyo mutuo, transforman los recursos disponibles en resultados esperados y que sean obtenidos en forma oportuna y eficaz. En este sentido, Ander Egg (2008), considera, que las funciones principales de la ejecución de las acciones cooperativas, se orientan hacia "la realización de actividades y tareas planificadas, vigilar el proceso comparándolo con los 
planes, de manera que se hagan los ajustes necesarios y se mantenga un sistema de información" (pág. 69).

\section{Valoración Ambiental}

Las preocupaciones que actualmente presenta la humanidad tales como: el deterioro de la calidad de vida, debido en parte a la contaminación ambiental y a la explosión demográfica, entre otros, le dan mayor significación a la valoración ambiental del entorno educativo. De allí, su importancia para la sociedad, la cual está consciente de los problemas que afectan al ambiente y por ello buscan en forma desesperada soluciones para armonizar las relaciones del hombre con su medio.

En este sentido, la educación ambiente debe trascender la realidad de su aplicación que consiste a juicio de Martínez (2006), "en trabajar determinados aspectos de las ciencias naturales y la ecología, a fin de conocer el entorno más cercano y los problemas clásicos" (pág. 25),

Esta sección del trabajo de investigación como lo expresan los autores es de gran relevancia porque permite situar, dentro del contexto las ideas y planteamientos que se desean realizar. Para elaborar las bases teóricas de la investigación se sugiere considerar los siguientes aspectos: Ubicación del problema en un enfoque teórico determinado, relación entre la teoría y el objeto de estudio, posición de distintos autores sobre el problema u objeto de investigación, adopción de una postura por parte del investigador, la cual debe ser justificada.

\section{Marco metodológico}

Desde esta perspectiva, el estudio está orientado por los parámetros de la investigación cualitativa, la cual tiene como propósito contribuir con el desarrollo óptimo de prácticas educativas.

A tal efecto, Palella y Martins (2010)," los paradigmas son el producto 
de las creencias, valores y técnicas compartidas socialmente; se construyen en el tiempo y se estructuran en contextos determinados" (pág. 39). En tal sentido, este paradigma cualitativo está ligado a las perspectivas estructural y dialéctica porque centra su atención en comprender los significados que los sujetos infieren a las acciones y conductas sociales. Para ello se utiliza esencialmente técnicas basadas en el análisis del lenguaje, como pueden ser la entrevista, el grupo de discusión, la historia de vida, y las técnicas de creatividad social.

Asimismo, este paradigma busca la objetividad en el ámbito de los significados utilizando como criterio de evidencia el acuerdo intersubjetivo en el contexto educativo.

\subsection{Diseño de la investigación}

Con respecto al método de investigación utilizado en el estudio es el Investigación Acción Participante, el cual según Elliott (2005), la define como:

Una ciencia educativa crítica y atribuye a la reforma educacional los predicados de participativa y colaborativa: plantea una forma de investigación educativa concebida como análisis crítico que se encamina a la transformación de las prácticas educativas, de los entendimientos educativos y los valores educativos de las personas que intervienen en el proceso, así como de las estructuras sociales e institucionales que definen el marco de la actuación de dichas personas (pág. 184).

Fase 1. Diagnóstico:

La Etapa de Diagnóstico: Eisman y Otros (2005), La define como: "identificación de un área problemática o necesidades básicas que se quieren resolver. Ordenar, agrupar, disponer y relacionar los datos de acuerdo con los objetivos de la investigación, es decir, preparar la información a fin de proceder a su análisis e interpretación" (pág. 262). 
Por consiguiente, este estudio, contempló la detección del problema reflejado en los docentes, niños y niñas.

La Etapa de Planificación: explica que en esta fase: "cuando ya se sabe lo que pasa, se ha diagnosticado una situación hay que decidir qué se va a hacer. En el plan de acción se estudiarán y establecerán prioridades en las necesidades, y se harán opciones ente las posibles alternativas" (pág. 263). Por tanto, abarca el diseño y elaboración de un plan de acción, flexible y abierto a cambios o modificaciones que pudieran surgir durante la aplicación de cada actividad programada.

La Etapa de Ejecución: sostiene que: "es la actuación para poner el plan en práctica y la observación de sus efectos en el contexto en que tiene lugar" (pág. 263). Por ende, consistió en la puesta en práctica de las diversas acciones planificadas, cada una de las cuales se reflejan en la sistematización de actividades del presente estudio.

La Etapa de Evaluación: la definen como: "en torno a los efectos como base para una nueva planificación. Será preciso un análisis crítico sobre los procesos, problemas y restricciones que se han manifestado y sobre los efectos lo que ayudara a valorar la acción desde lo previsto, deseable y a sugerir un nuevo plan" (pág. 264). De ahí que esta fase permitirá verificar y comprobar que los contenidos, estrategias y herramientas que la investigadora programará conjuntamente con los niños y niñas, serán trabajados de manera adecuada y efectiva.

\subsection{Población y muestra:}

Eisman y Otros (2005) indica que "es una fuente importante y preliminar de información, dentro del cual tiene lugar el objeto de estudio" (pág. 173).

De allí que la presente investigación contará con la participación y colaboración de 31 informantes o participantes, conformado por cinco (5) docentes, veintidós (22) padres y representantes; así como veintiséis (26) 
niños y niñas del Centro de Educación Inicial Bolivariano "Cinqueña III" del municipio Barinas del estado Barinas.

\subsection{Validación}

Tomando en consideración el método acción participante, la validez según Pérez (2008) "concierne a la exactitud con que las conclusiones representen efectivamente la realidad empírica y si los constructos diseñados representan categorías reales de la experiencia humana" (pág. 80). De ahí que la validez depende de una actitud de aproximación de igual a igual, entre el evaluador y el evaluado. Es por ello que para llevar a cabo la validación de las informaciones se tomará en cuenta los métodos de triangulación, a través de los cuales, según Hernández Fernández, y Baptista (2010), expresan que la triangulación es el "hecho de utilizar las diferentes fuentes y métodos de recolección que permite integrar y contrastar toda la información disponible para construir una visión global, exhaustiva y detallada de una experiencia en particular" (pág. 622).

\subsection{Análisis de dato:}

Bajo esta premisa se inició el trabajo de la investigación por medio de la inducción para hacer un acercamiento del estudio, es decir, una interpretación partiendo de las informaciones aportadas por los docentes, padres y representantes en las entrevistas a profundidad; así como por los niños y niñas en guía de observación; de manera que se visualice el hecho en toda su expresión.

No obstante, para la realización de la triangulación de acuerdo a Rojas (2010) señala que "existen diversas modalidades o tipos de triangulación, pero para este estudio se considera la triangulación de fuentes" (pág. 166) la cual permite contrastar la información obtenida de diferentes sujetos o grupos de sujetos acerca de un tema. También se puede contrastar, por ejemplo, la 
información obtenida de personas y documentos. Cabe destacar la importancia de la triangulación es en el cruce entre los aspectos antes mencionados para dar mayor validez y fiabilidad a los resultados.

\subsection{Propuesta}

Siguiendo el orden de los planteamientos, se planificaron acciones, las cuales se dividieron a su vez en cuatro aspectos que fueron: El primero referido a la sensibilización sobre las acciones cooperativas que contribuyan a la valoración ambiental del entorno educativo; El segundo referente a la planificación de las actividades; el tercero concerniente al desarrollo de las actividades despertando el interés y motivación de los agentes involucrados, mejorando los aspectos relacionados con el acciones cooperativas que contribuyan a la valoración ambiental del entorno educativo; finalmente el último aspecto, una vez implementadas las acciones se hizo necesario la valoración de las acciones, por medio de una evaluación de impacto.

\section{Conclusiones}

En consideración a las acciones cooperativas que contribuyan en la valoración ambiental del entorno educativo se puede decir que el niño y niña en su etapa inicial está consolidando los esquemas, y es allí donde los principios van a regir el comportamiento del educando de manera social e individual; donde los valores ambientales son el pilar en la conducta conservacionista del individuo, porque no se puede concebir ningún proceso 0 sistema educativo que no desarrollen de manera clara y precisa los valores que orientan la valoración ambiental del entorno educativo. A pesar de ello, los actores en estudio están conscientes de la necesidad de poner en práctica acciones cooperativas en la institución, razón por la que la presente investigación busca interrelacionar el trabajo práctico con lo cognitivo. 
Para concluir, se afirma que las acciones cooperativas en el entorno educativo son necesarias y de gran valor en las instituciones, porque así lo manifestaron los docentes y adultos significativos de la institución. De acuerdo con las evidencias presentadas y las conclusiones se formulan las siguientes recomendaciones:

Continuar con la presente investigación partiendo de la evaluación del plan acción, donde el mismo deja ver el impacto ocasionado en el colectivo educativo.

Programar, jornadas de sensibilización sobre la valoración ambiental del entorno educativo y la importancia de los valores ambientales en los niños y niñas de educación inicial.

Coordinar en formación de colectivos hacia la acción del docente en relación a la valoración ambiental del entorno educativo.

Realizar seguimiento y control al comportamiento de los niños y niñas antes, durante y después del desarrollo de las acciones cooperativas; para revisar las ganancias en comportamiento ambiental del educando.

Solicitar ante la Zona educativa talleres y/o jornadas sobre valores ambientales y la valoración ambiental del entorno educativo.

\section{Referencias}

Cárdenas, E. (2007). Teoría y práctica de la educación. Caracas: Material mimeográfiado.

Eisman, L, Colas P. y Hernández, F. (2005). Métodos de Investigación en Psicopedagogía. España: McGraw-Hill.

Elliot, J. (2007). La Investigación-Acción en Educación. Madrid, España: Morata.

Grupo Ecológico Ozono (2006). Ecología y Ambiente. Revistas de Ambiente. Caracas. 
Hernández S, Fernández C. y Baptista, P. (2010). Metodología de la investigación. México. McGraw-Hill. Interamericana. Editores S.A.

Palella, S. y Martins F. (2010). Metodología de la Investigación Cuantitativa. Tercera Edición, Caracas. FEDUPEL.

Pérez, G. (2008). Investigación cualitativa: Retos e interrogantes. Madrid: La Muralla.

Rojas de E., B. (2010). Investigación cualitativa. fundamentos y praxis. 2da edición, Caracas: FEDUPEL. 


\section{Carmen Elena García Moreno \\ e-mail: jesmarbalni@gmail.com}

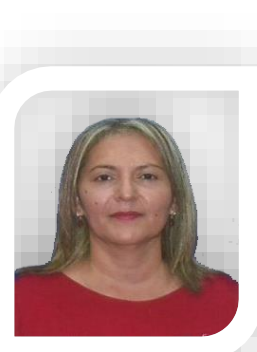

Licenciada en Educación Integral (UNELLEZ-Barinas), Especialista en Gerencia Educacional, UPELGuanare, Docente Titular en El C.E.I.B. "Cinqueña III", Participante en el curso de cuarto nivel de Formulación y Evaluación de Proyecto. 